\section{CPS-276 ATEZOLIZUMAB IN NON-SMALL CELL LUNG CANCER: EFFECTIVENESS AND SAFETY REAL WORLD DATA STUDY}

${ }^{1} \mathrm{JJ}$ Alcaraz Sanchez, ${ }^{2} \mathrm{JC}$ Del Rio Valencia, ${ }^{1} \mathrm{~A}$ Pintado Álvarez, ${ }^{1} \mathrm{~B}$ Mora Rodríguez, ${ }^{1} \mathrm{I}$ Muñoz Castillo*. 'Hospital Regional Universitario De Málaga, Hospital Pharmacy, Málaga, Spain; ${ }^{2}$ Hospital Xanit Benalmadena, Hospital Pharmacy, Benalmadena, Spain

10.1136/ejhpharm-2021-eahpconf.108

Background and importance Atezolizumab as monotherapy is indicated for the treatment of adult patients with locally advanced or metastatic non-small cell lung cancer (NSCLC) after prior chemotherapy. Patients with epidermal growth factor receptor (EGFR) mutant or anaplastic lymphoma kinase (ALK) positive NSCLC should also have received targeted therapies before receiving immunotherapy.

Aim and objectives To analyse the effectiveness and safety of treated patients with atezolizumab in usual clinical practice.

Material and methods An observational retrospective study was carried out between April 2018 and August 2020. Every patient with squamous and non-squamous NSCLC undergoing treatment with atezolizumab as monotherapy was included. Patient data were obtained from clinical records. Variables analysed were demographic variables (age and sex) and clinical variables (diagnosis, stage, treatment line, dose administered and performance status (PS) according to the ECOG scale). Efficacy endpoints were progression free survival (PFS) and overall survival (OS) assessed by iRECIST criteria and analysed by Kaplan-Meier curves. Adverse reactions were also assessed.

Results 35 patients were included, average age 63.52 \pm 11.25 years. $8.53 \%(n=3)$ had ECOG-PS 2 and the remainder had ECOG-PS 0-1 $(n=32)$. NSCLC stage was IV in $100 \%(n=35)$ of patients. $68.57 \%$ of patients started therapy with atezolizumab as secondline, $14.29 \%$ as thirdline and $17.14 \%$ as fourth/ fifth line. Only one patient had an EGFR positive mutation. The administered dose was $1200 \mathrm{mg} 3$ weekly. Four patients $(11.43 \%)$ were still receiving treatment. Causes of treatment suspension in the remaining patients were disease progression $(n=31)$, death $(n=4)$ or toxicity $(n=1)$. Median PFS was 3.2 months (95\% CI 2.6 to 7.2). Median OS was 6.3 months (95\% CI 4.4 to 9.1 ). $40 \%$ of patients received $\leq 3$ cycles of treatment.

Adverse reactions were: grade 2-3, asthenia 31.43\%, grade 1-2 arthralgia 14.29\%, anorexia $14.29 \%$, skin toxicity $17.14 \%$, gastrointestinal toxicity $28.57 \%$, pneumonitis $2.86 \%$, hepatic toxicity $8.57 \%$, rheumatological $14.29 \%$ and neurological $5.71 \%$. One case of gastrointestinal toxicity caused treatment suspension. Neither renal nor endocrine toxicity was recorded.

Conclusion and relevance Median PFS in our study was similar to that found in the OAK phase III trial (2.8 months). Atezolizumab was safe and well tolerated; the safety profile was similar to that described in clinical trials. $40 \%$ of patients receiving $\leq 3$ cycles could suggest hyperprogression in a high group of patients. Chemotherapy associated with immunotherapy needs to be studied in this no benefit subgroup of patients.

\section{REFERENCES AND/OR ACKNOWLEDGEMENTS}

Conflict of interest No conflict of interest

\section{CPS-277 CETUXIMAB VERSUS BEVACIZUMAB IN METASTATIC COLORECTAL CANCER: A COMPARATIVE EFFECTIVENESS AND PATIENT REPORTED OUTCOMES MULTI-COHORT STUDY}

\begin{abstract}
${ }^{1,2}{ }^{2}$ PP Marques*, ${ }^{3}$ AR Godinho, ${ }^{3}$ P Heudtlass, ${ }^{4} \mathrm{HL}$ Pais, ${ }^{4}$ A Quintela, ${ }^{1} \mathrm{JP}$ Lopes Da Cruz, ${ }^{2}$ AP Martins. ${ }^{1}$ Centro Hospitalar Universitário De Lisboa Norte-Hospital De Santa Maria, Pharmacy Department, Lisboa, Portugal; ' ${ }^{2}$ Faculty of Pharmacy of the University of Lisbon, Pharmacoepidemiology Department, Lisboa, Portugal; ${ }^{3}$ Centre for Health Evaluation and Research, Epidemiology Department, Lisboa, Portugal; ${ }^{4}$ Centro Hospitalar Universitário De Lisboa Norte-Hospital De Santa Maria, Medical Oncology Department, Lisboa, Portugal
\end{abstract}

\subsection{6/ejhpharm-2021-eahpconf.109}

Background and importance Uncertainty exists regarding the comparative effectiveness of cetuximab versus bevacizumab in metastatic colorectal cancer (mCRC) due to conflicting evidence of efficacy of previous randomised clinical trials and the absence of quality of life (HRQoL) studies in this setting.

Aim and objectives In order to assess clinical effectiveness and patient reported tolerability of the different targeted treatment options simultaneously, we conducted a mainly retrospective head-to-head multi-cohort study. The main retrospective study was designed to compare real world clinical outcomes from both antibodies. Concurrently, we nested in it a smaller prospective cohort study for the purpose of measuring patient reported outcomes (PROs).

Material and methods Retrospective cohorts were defined by treatment line, and subgroups by (K)RAS status and tumour sidedness. Among other effectiveness outcomes, we compared response rates, progression free survival (PFS) and overall survival (OS). PROs were measured prospectively through EORTC disease specific instruments. Methods and reporting followed STROBE guidelines and SISAQOL/SPIRIT-PRO recommendations.

Results Between 2010 and 2018, 311 mCRC patients were included in the overall analysis, of whom 44 were further allocated to PROs nested cohorts. Except for (K)RAS mutation status, baseline characteristics were balanced across groups. In the full analyses, PFS (firstline: $H R=0.85 ; \mathrm{p}=0.26$; secondline: $H R=1.16 ; p=0.51$ ) and $O S$ (firstline: $H R=0.83$; $\mathrm{p}=0.26$; secondline: $\mathrm{HR}=0.88 ; \mathrm{p}=0.58$ ) were similar between treatment arms. In subgroup analyses (firstline), we found a survival difference favouring bevacizumab in right-sided tumours (PFS: HR=0.52; $\mathrm{p}=0.025 ; \mathrm{OS}: \mathrm{HR}=0.60 ; \mathrm{p}=0.11$ ), but not in left-sided or (K)RAS wild-type tumours. Response rates were higher for bevacizumab in patients with right-sided primaries and similar across other comparisons. During the first 12 weeks of treatment, a higher proportion of patients in the cetuximab arm experienced clinically meaningful $(\geq 10 \%)$ deterioration of HRQoL comparing with the bevacizumab cohort: $53.8 \%$ versus $18.2 \%$ at 6 weeks and $66.7 \%$ versus $12.5 \%$ at 12 weeks. We also observed progressively increased scoring on the symptom scales in the cetuximab cohort during follow-up.

Conclusion and relevance This study provides evidence suggesting that bevacizumab and cetuximab containing regimens result in similar clinical effectiveness outcomes in mCRC, except for right-sided tumours, where bevacizumab performed substantially better. Cetuximab led to a progressive negative impact on HRQoL compared with baseline and bevacizumab. These findings should be further explored in randomised studies. 